\title{
Developing a Strategy for the Spatial Localisation and Autonomous Release of Silver Nanoparticles within Smart Implants
}

\author{
Laura A. A. Newton, ${ }^{1}$ Ray Leslie, ${ }^{1}$ and James Davis ${ }^{2}$ \\ ${ }^{1}$ School of Science and Technology, Nottingham Trent University, Nottingham NG11 8NS, UK \\ ${ }^{2}$ School of Engineering, NIBEC, University of Ulster, Jordanstown, Newtownabbey, Co. Antrim, Northern Ireland BT37 0QB, UK
}

Correspondence should be addressed to James Davis, james.davis@ulster.ac.uk

Received 1 May 2011; Accepted 10 May 2011

Academic Editor: Jay D. Wadhawan

Copyright ( $) 2011$ Laura A. A. Newton et al. This is an open access article distributed under the Creative Commons Attribution License, which permits unrestricted use, distribution, and reproduction in any medium, provided the original work is properly cited.

\begin{abstract}
The prevalence of antibiotic resistance has increased during the last few years and is viewed as a growing problem which has fuelled copious amounts of research on the development of new antibacterial agents. Silver is well recognized as possessing antibacterial activity, and so by harnessing these properties and incorporating silver nanoparticles (AgNPs) within microdevices, possessing microfluidic channels has much promise. Progress in developing these types of systems has been limited due to the technological difficulties involved in controlling the inclusion of these nanoparticles within the devices. This work provides an insight into a novel electrochemical interaction that can enable not only the localisation of silver within such structures but also enables the smart release of AgNPs.
\end{abstract}

\section{Introduction}

Access related infections (ARIs) are among the most prevalent causes of nosocomial infections and are complicated by the fact that bacteria associated with biofilm formation on the catheter surfaces play a key role in the morbidity and pathogenesis of these infections with hospital admissions for vascular ARIs having doubled in the last decade [1-4]. The bacteria contained within the biofilm layer benefit from having an increased resistance to antimicrobial agents and, as such, are generally more difficult to treat and to eradicate [5]. While systemic antibiotics are largely effective at eliminating circulating bacteria, their ability to sterilize lines in which a biofilm has taken hold is less efficient and life-threatening complications may arise. Most of these are treatable albeit at a price in terms of morbidity for the patient and financial costs incurred by the need for intravenous antibiotics and an increased length of hospital stay $[6,7]$. This is compounded by the alarming long-term issues associated with the repeated use of antibiotic regimes in such contexts as they have been attributed to the selection of multi-resistant organisms, such as methicillinresistant Staphylococcus aureus (MRSA) and vancomycin-resistant enterococci $[8,9]$.

There has been much research into surface modifications incorporating antibacterial components that attempt to minimise the propensity for biofilm formation. In spite of this, no currently available indwelling line is entirely free from the complication of infection. The incorporation of silver particles as an antibacterial coating is, however, now commonplace whereby its ionized form has been shown to bind to tissue proteins and bacterial RNA/DNA resulting in structural changes that can inhibit cell replication and can result in cell distortion and death [10-12]. Silver has long been viewed as an ideal weapon to be used in the fight against pathogenic bacteria, such that the impregnation of silver nanoparticles (AgNPs) within medical devices and wound dressings to prevent bacterial growth is routine [13-17]. Conventional approaches to the incorporation of silver are well suited for macroscale application, but their deployment within the emerging microscale devices creates an issue. There is also some concern over their mid- to long-term effectiveness as the coatings, irrespective of composition, 
can be rendered inactive by the exopolysaccharides that constitute the biofilm. Numerous investigations have shown silver-impregnated tubes to be ineffective at preventing the development of biofilms with the formation of the latter leading to capsule formation that could serve to promote reinfection [4]. It could be proffered that their main value lies more in slowing the development of the biofilm rather than in its prevention [3-5].

\section{Methodology}

Harnessing the antibacterial properties of AgNPs within microdevices possessing microfluidic channels that could be prone to bacterial colonisation creates a technological dilemma in terms of not only controlling their incorporation within the device but in producing the nanoparticle in a form that is readily accessible to the bacteria and thus maintains the antimicrobial action. AgNPs can be synthesized using a variety of different techniques, and at present, they are mainly produced by bulk processing methods, through the chemical reduction of a silver salt (typically silver nitrate), which allows them to be subsequently implanted within macrostructures [18, 19]. Electrochemical methods for preparing AgNPs and nanostructured surfaces have also been widely reported $[18,20]$, and while the present investigation seeks to adapt the basis of the former, the remit was focused more on a novel approach that could enable not only the localisation of silver within such structures but also the controlled formation of AgNPs which can be either localised at the device interface or ejected into the solution channel.

The basis of the approach lies in the functionalisation of an electrode surface with silver through conventional electrodeposition methods $[18,20]$. The surface is then oxidised to strip the silver, but importantly, this is done in the presence of a thiol ligand. The sulfur functionality binds to the released silver ion resulting in the formation of a silver thiolate complex (Ag-S-R). This is insoluble and is effectively deposited at the electrode surface. Electrochemical reduction of the silver-thiolate bond would, under normal instances, be expected to lead to the release of the thiol back into solution. Such transformations have long been studied-particularly with thiolate monolayers on gold [21, 22 ]. In such cases, the gold from the gold thiolate is simply reincorporated back within the underlying gold layer [22]. In this case, the normal expectation, and our original aim, would be for the silver to be redeposited onto polycrystalline platinum-ultimately leading to a nanostructured deposit on the surface. It was found, however, that, depending on the thiol used, a wholly different behaviour could be obtained, and rather than reintegration of the silver onto the platinum surface, electro-ejection of AgNPs can occur.

A variety of silver thiolate systems have been investigated in an effort to elucidate the underlying dynamics of the interfacial processes involved which enables the controlled ejection of AgNPs. These were facilitated through a combination of electroanalytical investigations, electrochemical quartz crystal microbalance (EQCM) studies, atomic force microscopy (AFM), electron microscopy (SEM-EDX), and $\mathrm{X}$-ray photoelectron spectroscopy (XPS).

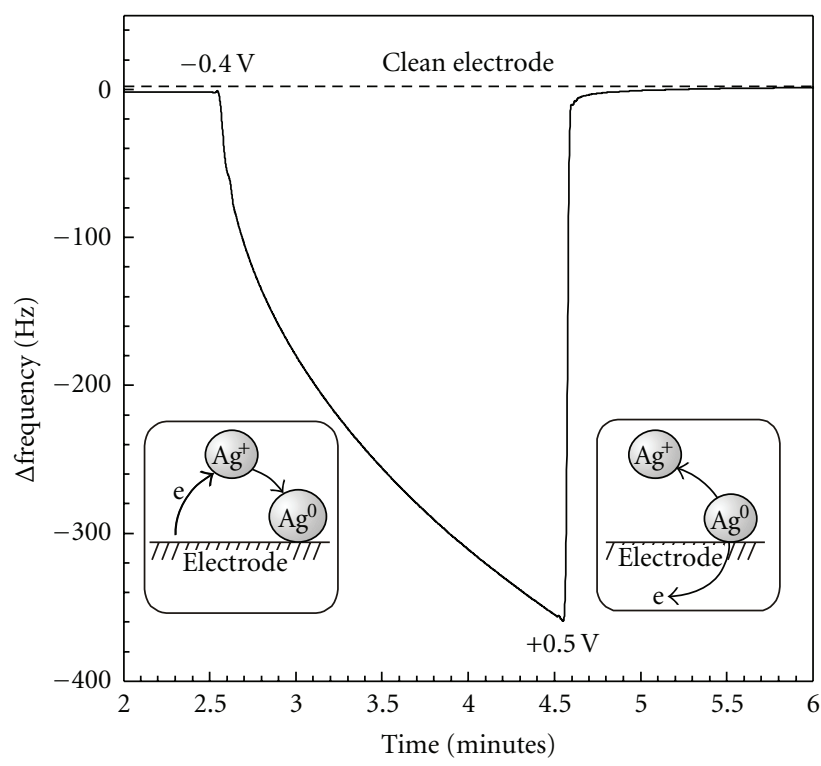

FIGURE 1: EQCM data highlighting the deposition and stripping of silver.

\section{Results}

The preliminary investigations were performed using an EQCM. The silver was electrodeposited onto a platinum crystal used as the working electrode $(-0.4 \mathrm{~V})$. It could then be stripped off from the surface through the application of an oxidising potential $(+0.5 \mathrm{~V})$ as indicated in Figure 1. The decrease in the frequency indicates the accumulation of silver at the surface, and the return to the baseline the subsequent removal of the silver ion into the bulk of the solution.

The process was repeated but with a view to assessing the impact of a thiol additive (homocysteine, $10 \mathrm{mM}$ ). Silver was electrodeposited $(-0.4 \mathrm{~V})$ onto the crystal as before from a simple solution of silver nitrate. The silverplated crystal was then removed, rinsed and placed in a buffered $(\mathrm{pH} 7)$ solution of the thiol and the electrode held at $0 \mathrm{~V}$ to equilibrate. The imposition of the oxidising potential $(+0.5 \mathrm{~V})$ resulted in a further decrease in the crystal frequency (Figure 2) and is in marked contrast to the behaviour observed in Figure 1.

This is attributed to the stripping of $\mathrm{Ag}^{0}$ to $\mathrm{Ag}^{+}$, the subsequent formation of the silver-thiolate complex (Ag-SR) and its deposition at the electrode as a consequence of the poor solubility of the resulting product. It was anticipated that the imposition of a reducing potential $(-1.0 \mathrm{~V})$ would therefore result in the reduction of the Ag-SR bond resulting in the release of the thiol but the retention of the silver at the electrode. Similar processes have been observed on gold substrates, and the initial behaviour followed this prediction with a sharp increase in crystal frequency. However, rather than simply releasing the thiol, the frequency returned to almost that observed with a clean platinised crystal indicating that the entire silver layer had been lost. 


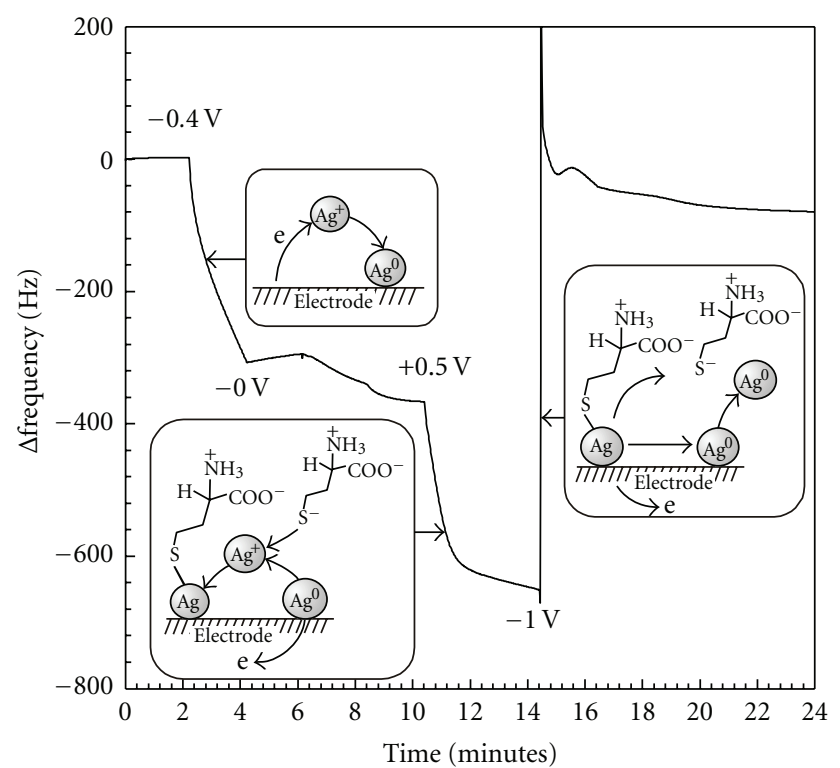

FIGURE 2: EQCM data highlighting the deposition and stripping of silver in the presence of homocysteine.

Visual examination of both the crystal and the interfacial solution layer revealed the appearance of colloidal silver aggregates. This was corroborated by conducting elemental analysis of the both the bulk solution and the solution adjacent to the electrode interface. This is clearly contrary to what would normally be expected. The release of silver into the solution is usually mandated through an oxidising potential (cf. Figure 1), whereas the reducing potential should have resulted in the electrodeposition of the silver consistent with the behaviour shown in Figure 1 and when repeating the above experiment in absence of the thiol. This therefore suggests that the properties of the amino acid thiol must be instrumental in removing the silver.

The experiment was repeated with 3-mercaptopropane, and the results are shown in Figure 3. The EQCM profile is initially similar to that obtained with the homocysteine, but there is a marked difference when the electroreduction of the Ag-SR complex is attempted. As expected, there is an increase in the frequency as the thiol is released back into solution, and it then returns to the value observed before the oxidative step was applied. In contrast to the profile observed with homocysteine, it effectively terminates at this point indicating that the silver is retained at the electrode-in keeping with what would normally be expected.

The experiments were repeated with 3-mercaptopropanol and the tripeptide, glutathione. The former was found to possess an EQCM profile almost identical to that of the propane analogue while glutathione mirrored that of the homocysteine-indicating that both of the previous responses were accurate but providing two distinct pathways which could be considered to be representative of the nature of the thiol used. The main difference between the two groups is the presence of the acid-base functionality. Both homocysteine and glutathione possess the latter.

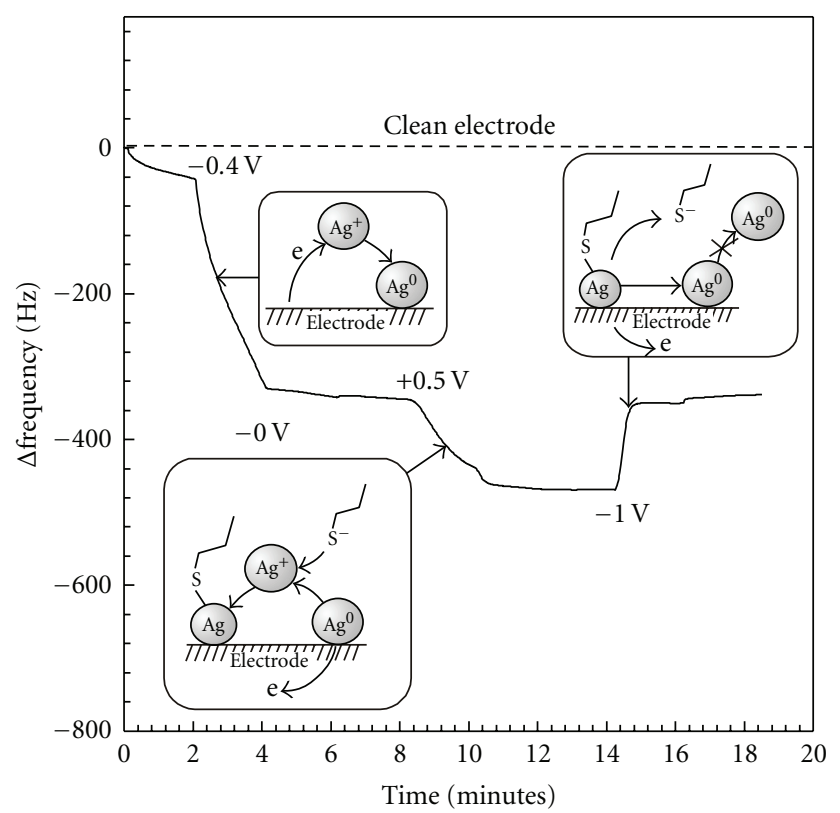

FIGURE 3: EQCM data highlighting the deposition and stripping of silver in the presence of 3-mercaptopropane.

\section{Discussion}

An initial assumption was that the complexing properties of the amino acid group must be responsible for the removal of the silver. However, this is flawed in two respects. The electrooxidation of the silver in the presence of the amino acid thiol leads to an insoluble product. The greater hydrophilicity inherent to glutathione was also found to be ineffectual in mobilising the product into solution. In addition, the reducing potential $(-1 \mathrm{~V})$ reduces the silver (I) to metallic silver thus making it unavailable for complex formation. It could be rationalised that the oxidation process leading to the $\mathrm{Ag}$ SR formation leads to the precipitation of a loose aggregation of Ag-SR particles. The subsequent reduction of the Ag-SR species at the immediate surface leads to the release of the thiol which then induces the release of the overlying layer. In this respect, the interfacial Ag-SR serves as electroreducible anchor. Yet, the mercaptopropane and mercaptopropanol results clearly contradict this hypothesis as the entire silver is effectively recaptured and only the thiol is released.

The presence of the acid-base groups appears pivotal to the release mechanism, but rather than through complexation, the mode of action could be through their ability to serve as conduits for the transfer of electroreducible protons to the electrode. The underlying platinum on the crystal is an excellent catalytic substrate for the electroreduction of protons, and given the application of the reducing potential $(-1 \mathrm{~V})$, it could well be that the adherence of the metallic silver (or aggregate layer) is weakened as a consequence of the formation of an adsorbed hydrogen adlayer on the platinum.

\section{Conclusions}

The results highlight the tunable nature of the electrodeposited Ag-SR interaction. While it is clear that it is possible 
to control the physical ejection of the Ag-SR layer through the application of an appropriate potential, the nature of the ligand composition is shown to be crucial. The electrochemical approach provides an option for controlling not only the spatial location of the Ag-SR particles/aggregations but also enables control over their release.

\section{References}

[1] K. A. Barroclough, C. M. Hawley, G. Playford, and D. W. Johnson, "Pevention of access-related infection indialysis," Expert Review of Antiinfective Therapy, vol. 7, no. 10, pp. 11851200, 2009.

[2] E. L. Lawrence and I. G. Turner, "Materials for urinary catheters: a review of their history and development in the UK," Medical Engineering and Physics, vol. 27, no. 6, pp. 443453, 2005.

[3] F. Suska, S. Svensson, A. Johansson et al., "In vivo evaluation of noble metal coatings," Journal of Biomedical Materials Research, vol. 92, no. 1, pp. 86-94, 2010.

[4] E. Hurrell, E. Kucerova, J. Caubilla-Barron, and S. J. Forsythe, "Biofilm formation on enteral feeding tubes by Cronobacter sakazakii, Salmonella serovars and other Enterobacteriaceae," International Journal of Food Microbiology, vol. 136, no. 2, pp. 227-231, 2009.

[5] M. E. Falagas, A. M. Kapaskelis, V. D. Kouranos, O. K. Kakisi, Z. Athanassa, and D. E. Karageorgopoulos, "Outcome of antimicrobial therapy in documented biofilm-associated infections: a review of the available clinical evidence," Drugs, vol. 69, no. 10, pp. 1351-1361, 2009.

[6] C. Stephens, "Microbiology: breaking down biofilms," Current Biology, vol. 12, no. 4, pp. R132-R134, 2002.

[7] C. A. Fux, J. W. Costerton, P. S. Stewart, and P. Stoodley, "Survival strategies of infectious biofilms," Trends in Microbiology, vol. 13, no. 1, pp. 34-40, 2005.

[8] K. Hiramatsu, L. Cui, M. Kuroda, and T. Ito, "The emergence and evolution of methicillin-resistant Staphylococcus aureus," Trends in Microbiology, vol. 9, no. 10, pp. 486-493, 2001.

[9] G. A. Noskin, "Vancomycin-resistant enterococci: clinical, microbiologic, and epidemiologic features," Journal of Laboratory and Clinical Medicine, vol. 130, no. 1, pp. 14-20, 1997.

[10] M. Rai, A. Yadar, and A. Gadi, "Silver nanoparticles as a new generation of antimicrobials," Biotechnology Advances, vol. 27, no. 1, pp. 76-83, 2009.

[11] V. K. Sharma, R. A. Yngard, and Y. Lin, "Silver nanoparticles: green synthesis and their antimicrobial activities," Advances in Colloid and Interface Science, vol. 145, no. 1-2, pp. 83-96, 2009.

[12] J. S. Kim, E. Kuk, K. N. Yu et al., "Antimicrobial effects of silver nanoparticles," Nanomedicine: Nanotechnology, Biology, and Medicine, vol. 3, no. 1, pp. 95-101, 2007.

[13] J. M. Schierholz, L. J. Lucas, A. Rump, and G. Pulverer, "Efficacy of silver-coated medical devices," Journal of Hospital Infection, vol. 40, no. 4, pp. 257-262, 1998.

[14] D. R. Monterio, L. F. Gorup, A. S. Takamiug et al., "The growing importance of materials the prevent microbial adhesion: antimicrobial effects of medical devices containing silver," International Journal of Antimicrobial Agents, vol. 34, no. 2, pp. 103-110, 2009.

[15] M. E. Rupp, T. Fitzgerald, N. Marion et al., "Effect of silver-coated urinary catheters: efficacy, cost-effectiveness, and antimicrobial resistance," American Journal of Infection Control, vol. 32, no. 8, pp. 445-450, 2004.
[16] S. Gaisford, A. E. Beezer, A. H. Bishop, M. Walker, and D. Parsons, "An in vitro method for the quantitative determination of the antimicrobial efficacy of silver-containing wound dressings," International Journal of Pharmaceutics, vol. 366, no. 1-2, pp. 111-116, 2009.

[17] M. A. A. O’Neill, G. J. Vine, A. E. Beezer et al., "Antimicrobial properties of silver-containing wound dressings: a microcalorimetric study," International Journal of Pharmaceutics, vol. 263, no. 1-2, pp. 61-68, 2003.

[18] W. Zhang, X. Qiao, and J. Chen, "Synthesis of silver nanoparticles-effects of concerned parameters in water/oil microemulsion," Materials Science and Engineering B, vol. 142, no. 1, pp. 1-15, 2007.

[19] J. H. Lim and J. S. Lee, "A statistical design and analysis illustrating the interactions between key experimental factors for the synthesis of silver nanoparticles," Colloids and Surfaces $A$, vol. 322, no. 1-3, pp. 155-163, 2008.

[20] M. Mazur, "Electrochemically prepared silver nanoflakes and nanowires," Electrochemistry Communications, vol. 6, no. 4, pp. 400-403, 2004.

[21] M. B. Buchmann, T. Fyles, and T. Sutherland, "Electrochemical release from gold-thiolate electrodes for controlled insertion of ion channels into bilayer membranes," Bioorganic and Medicinal Chemistry, vol. 12, no. 6, pp. 1315-1324, 2004.

[22] D. F. Yang and M. Morin, "Chronoamperometric study of the reduction of chemisorbed thiols on Au(111)," Journal of Electroanalytical Chemistry, vol. 429, no. 1-2, pp. 1-5, 1997. 


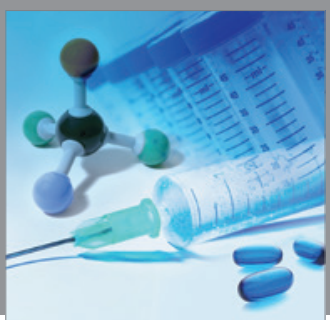

International Journal of

Medicinal Chemistry

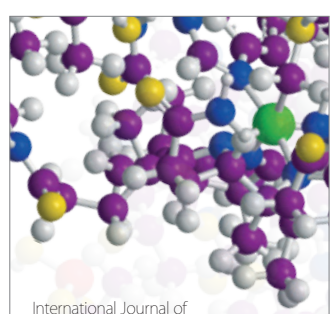

Carbohydrate Chemistry

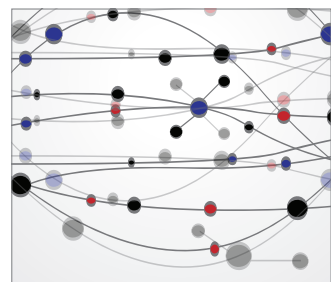

The Scientific World Journal
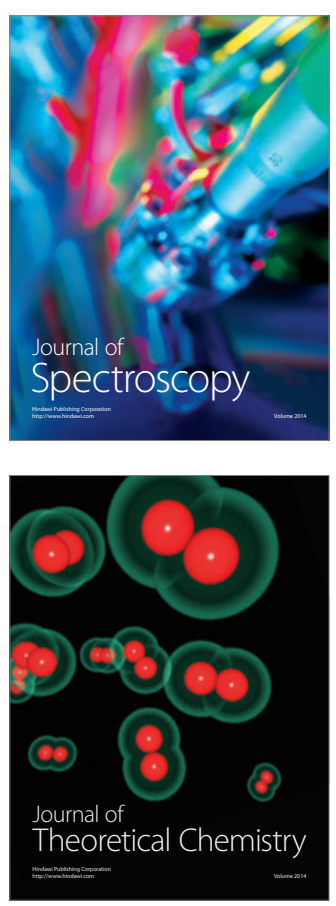
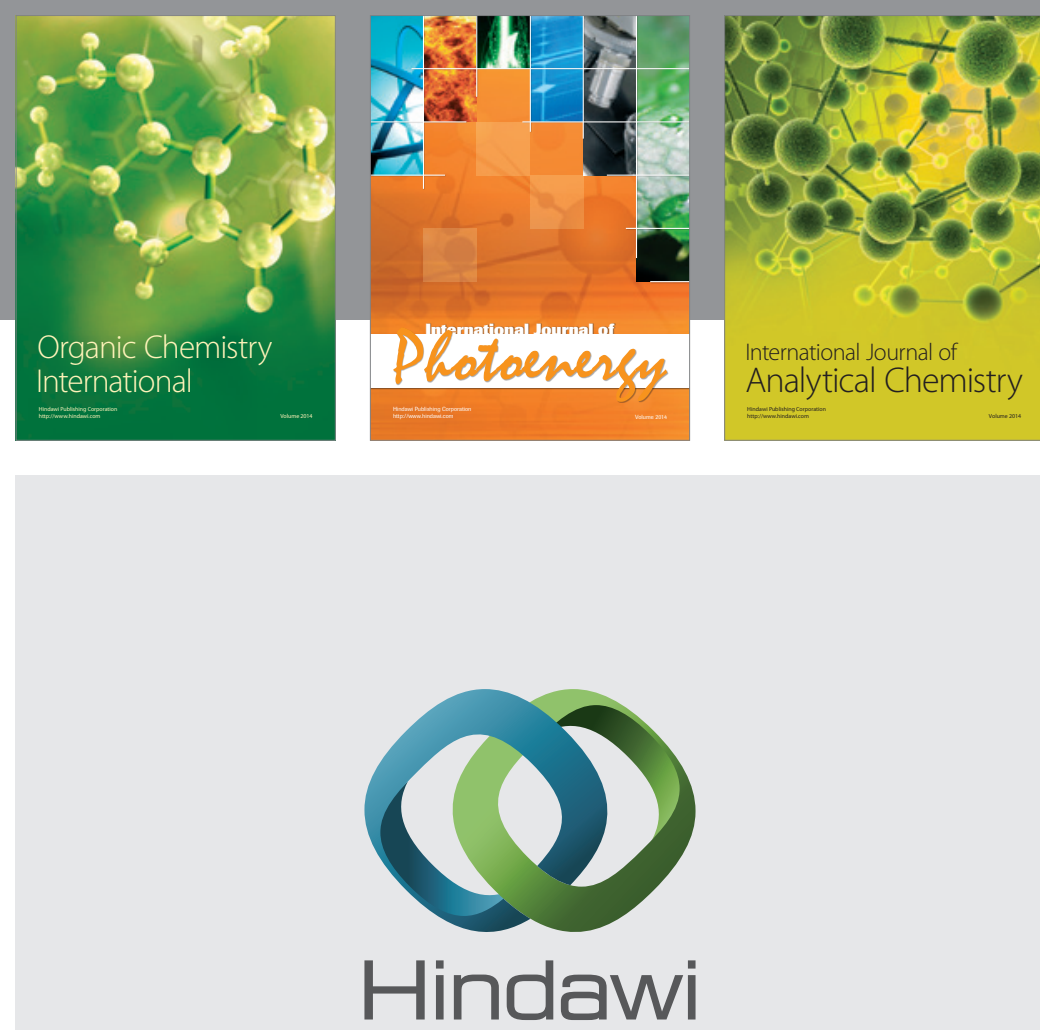

Submit your manuscripts at

http://www.hindawi.com
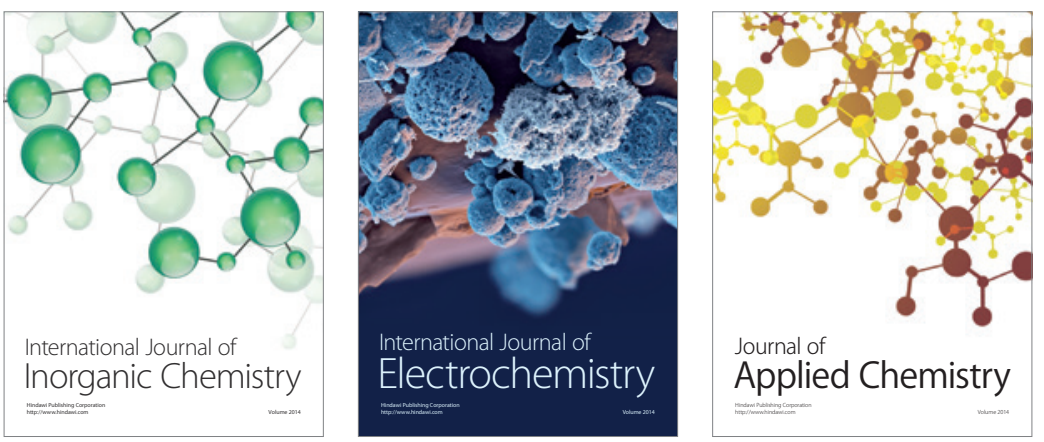

Journal of

Applied Chemistry
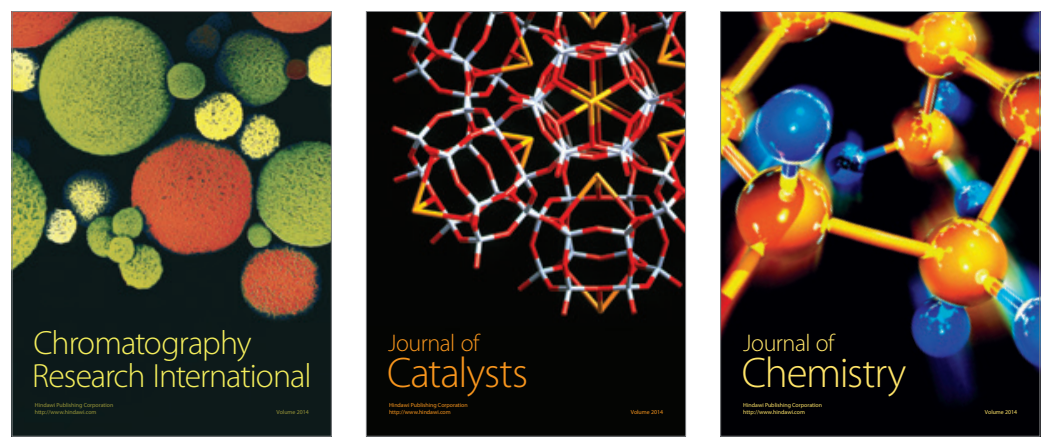
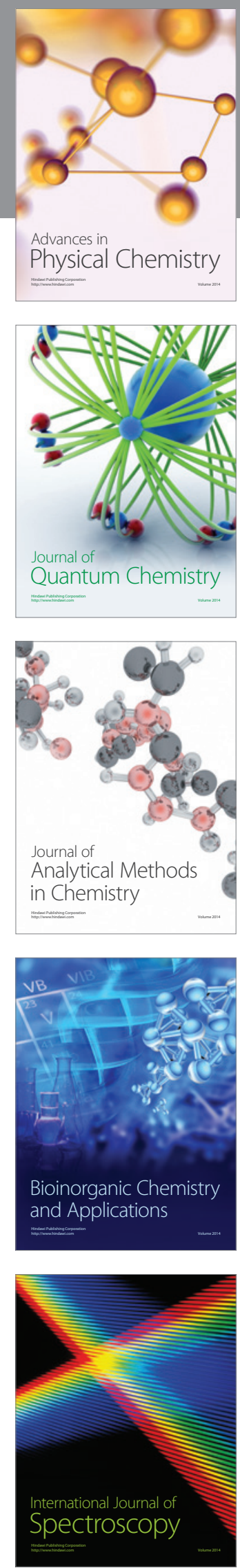